\title{
MS03-P13 | PROFILINS AND THE QUEST FOR STRUCTURE-BASEd IgE-EPITOPE MAPPING
}

Wortmann, Judith (Institute for Molecular Biosciences, University of Graz, Graz, AUT); Gottstein, Nina Alice (Institute for Molecular Biosciences, University of Graz, Graz, AUT); Hofer, Gerhard (University of Graz, Graz, AUT); Keller, Walter (Institute for Molecular Biosciences, University of Graz, Graz, AUT)

Background: Panallergens frequently cause respiratory allergy and oral allergy syndrome. Profilins are important panallergens because of their highly conserved structure and ubiquitous presence in allergy sources. Therefore, determination of the structure and biophysical characterization are important tasks to complement IgE-binding studies and enable structure-based epitope prediction. Here we give an overview of important profilin allergens and present our most current research results.

Methods: Six profilins were expressed in E.coli. The food allergen Cuc m 2 (melon - Cucumis melo) and Cit s 2 (orange - Citrus sinensis), the pollen allergen Ole e 2 (olive tree - Olea europaea), Fra e 2 from the European ash (Fraxinus excelsior) and Phl p 12 (timothy grass - Phleum pratense) as well as the storage mite allergen Tyr p 36 (Tyrophagus puturescentiare). Biophysical characterization including Circular Dichroism spectroscopy, Differential Scanning Fluorimetry and Size Exclusion Chromatography are used to verify the proteins' native fold. Crystallization and homology modeling coupled to bioinformatics provide structural information and IgE-binding assays immunological data on cross-reactivity.

Results: All six profilin allergens included in this study were recombinantly expressed and purified to homogeneity. Biophysical characterization revealed the structural integrity of the allergens and their monomeric state, which makes them well suited for immunological testing. Homology modeling elucidated the surface-accessible regions of the allergens.

Conclusion: Immunological data regarding cross-reactivity of a wide range of homologous proteins in combination with structural information will enable more accurate IgE-epitope predictions using a structure based IgE-epitope mapping approach for the profilin family of allergens. 